\title{
La bronchite chronique chez les Autochtones - prévalence et facteurs associés
}

\author{
S. Konrad, M. Sc. (1); A. Hossain, M. Sc. (1); A. Senthilselvan, Ph. D. (2); J. A. Dosman, M.D. (3); P. Pahwa, Ph. D. (1, 3)
}

Cet article a fait l'objet d'une évaluation par les pairs.

\section{Résumé}

Introduction : On sait peu de choses sur la bronchite chronique (BC) chez les Autochtones au Canada. Le but de cette étude était de déterminer la prévalence de la BC et des facteurs qui lui sont associés chez les Autochtones de 15 ans et plus.

Méthodologie : Une analyse de régression logistique a été appliquée à des données tirées de l'Enquête auprès des peuples autochtones de 2006 (enquête transversale) afin de déterminer les facteurs de risque associés à la BC.

Résultats : La prévalence de la BC était de 6,6\% au sein des membres des Premières nations, de 6,2 \% chez les Métis et de 2,4 \% chez les Inuits. Elle était plus élevée chez les femmes que chez les hommes (7,2 \% contre 5,0\%). Les individus atteints de BC étaient en général plus âgés et plus nombreux à avoir un revenu et un niveau d'instruction plus faibles et à habiter en milieu rural. Le tabagisme et l'indice de masse corporelle étaient également associés de façon significative à la $\mathrm{BC}$, mais leur effet différait selon le sexe. L'obésité était associée de manière particulièrement significative à la BC chez les femmes, et le fait d'être fumeur ou de n'avoir jamais fumé était aussi associé de façon significative à la BC chez les femmes.

Conclusion : Ces constatations permettent de déterminer les facteurs associés à la BC chez les Autochtones. Ce sont peut-être à ce titre des facteurs de risque potentiellement évitables qui peuvent éclairer les pratiques en matière de promotion de la santé et de prévention des maladies.

Mots-clés : bronchite chronique, Autochtones, Enquête auprès des peuples autochtones

\section{Introduction}

Les Autochtones - membres des Premières nations, Métis et Inuits - sont en nettement plus mauvaise santé que la population canadienne en général ${ }^{1}$, une tendance également observée sur le plan respiratoire $^{2}$. Selon l'Enquête sur la santé dans les collectivités canadiennes (ESCC) de $2005^{3}$, environ $15 \%$ des Autochtones ont reçu un diagnostic de l'une au moins de quatre maladies respiratoires (asthme, bronchite chronique $[\mathrm{BC}]$, emphysème et maladie pulmonaire obstructive chronique
[MPOC]), contre $10 \%$ de la population canadienne non autochtone. En 2000, les taux de congés d'hôpital normalisés selon l'âge dans l'Ouest du Canada pour toutes les maladies respiratoires étaient chez les Autochtones de 3040 pour 100000 habitants, contre 920 pour $100000 \mathrm{au}$ sein de la population non autochtone . $^{4}$

La BC est une maladie respiratoire définie ainsi : " Toux avec expectorations pendant au moins trois mois de l'année, pendant au moins deux ans ${ }^{5}$ ». La BC est une cause de morbidité importante et une maladie sous-jacente prédisposant à une MPOC $^{6}$.

On sait peu de choses sur la BC chez les Autochtones au Canada. L'Enquête régionale longitudinale sur la santé des Premières nations de 2002-2003 conclut à une prévalence normalisée selon l'âge de la BC autodéclarée comme diagnostiquée par un médecin de 3,7 \% chez les membres des Premières nations vivant dans des réserves ${ }^{7}$; la prévalence chez les Autochtones vivant hors réserve est de $4,9 \%$. Ces deux taux sont supérieurs à la prévalence au sein de la population canadienne non autochtone, qui est de $2,4 \%$, selon l'ESCC de $2005^{3}$.

La prévalence de la BC chez les Autochtones est peut-être élevée à cause de la forte prévalence de divers facteurs de risque. Le tabagisme, un faible revenu familial, un faible niveau d'instruction et des logements insalubres, souvent associés de façon significative à la prévalence et à l'incidence de la $\mathrm{BC}^{8-10}$, sont plus prévalents chez les Autochtones. Selon l'Enquête régionale longitudinale sur la santé des Premières nations de 2002-2003, environ 59 \% des membres des Premières nations déclaraient fumer, les taux de tabagisme étant plus élevés chez ceux qui vivaient dans des réserves que chez les autres $^{7}$. Les taux de tabagisme chez les Inuits atteindraient jusqu'à $70 \%{ }^{11}$.

En 2005, le revenu total médian des Autochtones de 25 à 54 ans (22 000 \$) était nettement inférieur à celui de la population non autochtone d'âge identique $(33000 \$)^{12}$. Dans le groupe des 25 à 64 ans, $44 \%$ des Autochtones (contre $60 \%$

Rattachement des auteurs :

1. Département de santé communautaire et d'épidémiologie, Université de la Saskatchewan, Saskatoon (Saskatchewan), Canada

2. Département des sciences de la santé publique, École de santé publique, Université de l'Alberta, Edmonton (Alberta), Canada

3. Centre canadien de santé et sécurité en milieu agricole, Université de la Saskatchewan, Saskatoon (Saskatchewan), Canada

Correspondance : Punam Pahwa, Centre canadien de santé et sécurité en milieu agricole, Université de la Saskatchewan, 103, Hospital Drive, Saskatoon (Saskatchewan) S7N 0W8; tél. : 306-966-7944; téléc. : 306-966-7920; courriel : pup165@mail.usask.ca 
de l'ensemble de la population) avaient fait des études postsecondaires ${ }^{13}$. Or un niveau d'instruction inférieur est souvent associé à un statut socioéconomique inférieur, ce qui peut aller de pair avec un revenu inférieur et de pires conditions de logement. En 2006, les Autochtones étaient pratiquement quatre fois plus nombreux à vivre dans des logements surpeuplés et trois fois plus nombreux à vivre dans un logement nécessitant des réparations importantes que les Canadiens non autochtones $^{14}$. Or qui dit mauvaises conditions de logement dit souvent humidité et moisissures, ce qui peut entraîner des problèmes respiratoires ${ }^{2}$.

Nous avons procédé à une étude descriptive afin d'évaluer la relation entre la BC et les caractéristiques personnelles et contextuelles. Les déterminants de la BC chez les Autochtones du Canada n'étant pas encore bien établis, cette étude visait à confirmer la prévalence (brute et ajustée) de la $\mathrm{BC}$ et à en déterminer les facteurs connexes chez les Autochtones canadiens de 15 ans et plus vivant hors réserve.

\section{Méthodologie}

\section{Population étudiée et source des données}

L'Enquête auprès des peuples autochtones (EAPA) de 2006 est une enquête transversale nationale réalisée entre octobre 2006 et mars 2007 par Statistique Canada en partenariat avec des organisations autochtones $^{15}$. C'était la troisième fois que Statistique Canada la réalisait, la première datant de 1991 et la deuxième de 2001 . Elle avait pour population cible les membres des Premières nations vivant hors réserve, les Métis et les Inuits vivant en milieu urbain, rural et dans le Nord dans l'ensemble du Canada. Un plan d'échantillonnage à plusieurs degrés a été utilisé pour choisir et recueillir des données de toutes les provinces. On peut trouver ailleurs des détails sur ce plan d'échantillonnage ${ }^{15}$. En bref, un échantillon cible a été créé à partir des réponses positives à quatre questions de sélection du questionnaire de recensement complet de 2006 indiquant que les répondants soit avaient des ancêtres autochtones, soit s'identifiaient comme Indiens de l'Amérique du
Nord, Métis ou Inuits, soit étaient Indiens inscrits ou relevaient des traités, soit étaient membres d'une bande indienne, soit une combinaison de ces réponses. L'échantillon a ensuite été divisé selon les domaines d'estimation et en fonction de l'identité autochtone, des groupes d'âge et des zones géographiques. Un échantillon aléatoire a alors été choisi pour chaque domaine d'estimation. L'EAPA comprenait des renseignements sur l'identité et l'ascendance autochtone, l'instruction, la langue, l'activité professionnelle, le revenu, la santé, les technologies des communications, la mobilité, le logement et le milieu familial. On a sélectionné en tout 48921 participants, avec un taux de réponse de 80,1 \%. Les données ont été recueillies au moyen de questionnaires autoremplis ou d'entrevues personnelles réalisées au téléphone ou en face à face.

Les populations cibles de cette enquête étaient les jeunes et les enfants autochtones (de 6 à 14 ans) et les adultes autochtones (15 ans et plus). Comme notre étude portait essentiellement sur la population adulte, nous avons exclu les participants à l'EAPA âgés de moins de 15 ans.

Le comité d'éthique de la recherche de l'Université de la Saskatchewan a approuvé ces recherches. Nous avons été autorisés à accéder aux données de Statistique Canada et avons effectué toutes les analyses au Centre de données de recherche de Statistique Canada à l’Université de la Saskatchewan.

\section{Mesures}

L'EAPA comprenait une série de questions destinées à connaître les maladies chroniques des participants à l'enquête. Les variables utilisées dans l'analyse sont définies ci-dessous.

\section{Résultat}

Dans ce rapport ${ }^{15}$, la variable de résultat nous intéressant pour les adultes reposait sur la question suivante : "Est-ce qu'un médecin, une infirmière ou un autre professionnel de la santé vous a dit que vous souffriez de bronchite chronique? »

\section{Facteurs}

Les variables démographiques et celles du milieu de vie, ainsi que les variables relatives à la santé et au mode de vie, présentaient un intérêt (voir le tableau 1). Les variables démographiques comprenaient l'âge, le sexe, l'origine ethnique et la situation de famille; les variables du milieu de vie comprenaient le lieu de résidence (urbain/rural), le nombre de personnes par ménage et la zone géographique. Le lieu de résidence reposait sur les définitions de Statistique Canada (peuplement et densité de population minimaux par $\mathrm{km}^{2}$ ) et les zones géographiques étaient les Territoires (Yukon, Territoires du Nord-Ouest et Nunavut), la ColombieBritannique, les Prairies (Alberta, Saskatchewan et Manitoba), l'Ontario, le Québec et les provinces de l'Atlantique (Nouveau-Brunswick, Île-du-PrinceÉdouard, Nouvelle-Écosse et TerreNeuve-et-Labrador). Les variables relatives à la santé comprenaient l'état de santé général autoévalué, le tabagisme et l'indice de masse corporelle (IMC). L'IMC a été adopté comme variable continue dans le modèle multivarié et a ensuite été divisé en classes pour une représentation schématique (figure 2). Les variables socioéconomiques comprenaient le niveau d'instruction et le revenu.

\section{Analyse statistique}

Nous avons calculé le pourcentage de participants déclarant souffrir de BC et mesuré les facteurs associés. Les variables de poids calculées par les spécialistes en méthodologie de Statistique Canada utilisées dans toutes les analyses ont garanti que les estimations finales seraient représentatives de la population étudiée. Nous avons utilisé une modélisation par régression logistique multiple pondérée et fondée sur une probabilité maximale afin de tester l'association des facteurs de risque de la BC. Une technique de rééchantillonnage avec répliques équilibrées répétées a été employée pour le calcul des erreurs types des coefficients de régression afin de tenir compte des regroupements hérités du plan d'étude de l'enquête transversale complexe. Les interactions réciproques statistiquement significatives ont été analysées. Les résultats des modèles sont 


\section{TABLEAU 1}

Caractéristiques des Autochtones (de 15 ans et plus) stratifiées selon l'autodéclaration de bronchite chronique, 2006, Canada ( $\mathrm{N}=48$ 921)

\begin{tabular}{|c|c|c|c|}
\hline & \multicolumn{2}{|c|}{ Bronchite chronique (\%) } & \multirow[t]{2}{*}{ RC (IC à $95 \%)$} \\
\hline & Oui & Non & \\
\hline \multicolumn{4}{|l|}{ Caractéristiques démographiques } \\
\hline \multicolumn{4}{|l|}{ Origine ethnique autochtone $\mathrm{a}^{\mathrm{a}}$} \\
\hline Indien de l'Amérique du Nord & 6,57 & 93,43 & 1,00 \\
\hline Métis & 6,19 & 93,81 & $0,93(0,79$ à 1,11$)$ \\
\hline Inuit & 2,38 & 97,62 & $0,35(0,25$ à 0,47$)$ \\
\hline \multicolumn{4}{|l|}{ Sexe } \\
\hline Homme & 5,00 & 95,00 & 1,00 \\
\hline Femme & 7,20 & 92,80 & $1,47(1,23$ à 1,76$)$ \\
\hline \multicolumn{4}{|l|}{ Âge (en années) } \\
\hline $15-19$ & 2,67 & 97,33 & 1,00 \\
\hline $20-24$ & 3,12 & 96,88 & $1,17(0,73$ à 1,86$)$ \\
\hline $25-34$ & 3,70 & 96,30 & $1,40(0,95$ à 2,06$)$ \\
\hline $35-44$ & 6,12 & 93,88 & $2,38$ (1,67 à 3,38$)$ \\
\hline $45-54$ & 9,09 & 90,91 & $3,64(2,57$ à 5,17$)$ \\
\hline 55 et plus & 10,06 & 89,94 & $4,07(2,83$ à 5,86$)$ \\
\hline \multicolumn{4}{|l|}{ Situation de famille } \\
\hline Marié & 6,85 & 93,15 & 1,00 \\
\hline Jamais marié & 4,28 & 95,72 & $0,61(0,50$ à 0,74$)$ \\
\hline Veuf ou divorcé & 10,59 & 89,41 & $1,61(1,30$ à 2,00$)$ \\
\hline \multicolumn{4}{|l|}{ Caractéristiques du milieu de vie } \\
\hline \multicolumn{4}{|l|}{ Nombre de personnes par ménage } \\
\hline$\geq 5$ & 4,08 & 95,92 & 1,00 \\
\hline $3-4$ & 5,32 & 94,68 & $1,34(1,01$ à 1,71$)$ \\
\hline$\leq 2$ & 8,22 & 91,78 & $2,11(1,63$ à 2,72$)$ \\
\hline \multicolumn{4}{|l|}{ Lieu de résidence $^{\mathrm{b}}$} \\
\hline Urbain & 6,61 & 93,39 & 1,00 \\
\hline Rural & 5,19 & 94,81 & $0,77(0,66$ à 0,91$)$ \\
\hline \multicolumn{4}{|l|}{ Zone géographique } \\
\hline Territoires $^{c}$ & 1,85 & 98,15 & 1,00 \\
\hline Colombie-Britannique & 4,95 & 95,05 & $2,78(1,94$ à 3,98$)$ \\
\hline Prairies $^{d}$ & 4,96 & 95,04 & 2,78 (2,05 à 3,78) \\
\hline Ontario & 9,05 & 90,95 & $5,31(3,82$ à 7,37$)$ \\
\hline Québec & 6,89 & 93,11 & $3,95(2,75$ à 5,66$)$ \\
\hline Provinces de l'Atlantique ${ }^{\mathrm{e}}$ & 7,44 & 92,56 & 4,29 (3,08 à 5,97) \\
\hline \multicolumn{4}{|l|}{ Statut socioéconomique } \\
\hline \multicolumn{4}{|l|}{ Niveau d'instruction } \\
\hline Diplôme universitaire & 3,93 & 96,07 & 1,00 \\
\hline Études universitaires & 6,82 & 93,18 & $1,78(1,31$ à 2,43$)$ \\
\hline Secondaire terminé & 5,29 & 94,71 & $1,36(0,94$ à 1,98$)$ \\
\hline Moins que le secondaire & 6,95 & 93,05 & $1,82(1,33$ à 2,49$)$ \\
\hline \multicolumn{4}{|l|}{ Revenu annuel (\$) } \\
\hline 100000 et plus & 2,69 & 97,31 & 1,00 \\
\hline $80000-99999$ & 3,88 & 96,12 & $1,46(1,01$ à 2,09$)$ \\
\hline $60000-79999$ & 5,71 & 94,29 & $2,19(1,58$ à 3,02$)$ \\
\hline
\end{tabular}

Suite page suivante présentés sous forme de rapports de cotes (RC) assortis d'intervalles de confiance (IC) à $95 \%$. Les progiciels statistiques SAS version 9.2 (SAS Institute Inc., Cary, Caroline du Nord, É.-U.) et STATA version 11.0 ont été utilisés pour toutes les analyses.

\section{Résultats}

Parmi les répondants adultes à l'EAPA, $50,0 \%$ étaient membres des Premières nations, 45,2 \% Métis et les autres, soit $4,8 \%$, Inuits. En raison de leur faible effectif au sein de l'ensemble de données, les Inuits ont été exclus de toutes les analyses multivariées.

\section{Prévalence brute de la bronchite chronique}

Le tableau 1 résume la prévalence et le rapport de cotes pour la BC. La prévalence brute de la BC était de 6,6 \% chez les membres des Premières nations, 6,2 \% chez les Métis et 2,4\% chez les Inuits, respectivement (tableau 1 ). La prévalence globale était de $6,0 \%$ chez les Autochtones vivant hors réserve. Elle était de $8,3 \%$ chez les fumeurs et de 3,3\% chez les non-fumeurs n'ayant jamais fumé. La BC était plus fréquente chez les femmes que chez les hommes (5,0 \% contre $7,2 \%$ ) et elle augmentait avec l'âge, passant de $2,7 \%$ chez les 15 à 19 ans à 10,1 \% chez les 55 ans et plus. C'est en Ontario $(9,1 \%)$ et dans les provinces de l'Atlantique $(7,4 \%)$ que la prévalence était la plus élevée. Elle était également plus forte chez ceux vivant avec un plus faible revenu et dont le niveau d'instruction était inférieur.

La prévalence était de 13,2 \% chez les diabétiques et de 8,1 \% chez les nondiabétiques.

\section{Prévalence ajustée de la bronchite chronique}

Le tableau 2 synthétise les variables qui se sont révélées être prédictrices importantes de la $\mathrm{BC}$ dans le modèle multivarié.

Dans le modèle multivarié, la prévalence de la BC chez les Métis ne différait pas de manière significative de la prévalence chez les Premières nations $(\mathrm{RC}=1,05$; 
TABLEAU 1 (Suite)

Caractéristiques des Autochtones (de 15 ans et plus) stratifiées selon l'autodéclaration de bronchite chronique, 2006, Canada ( $\mathrm{N}=48$ 921)

\begin{tabular}{|c|c|c|c|}
\hline & \multicolumn{2}{|c|}{ Bronchite chronique (\%) } & \multirow[t]{2}{*}{ RC (IC à 95 \%) } \\
\hline & Oui & Non & \\
\hline $40000-59999$ & 6,46 & 93,54 & $2,49(1,83$ à 3,40$)$ \\
\hline $20000-39999$ & 7,08 & 92,92 & 2,75 (2,05 à 3,69) \\
\hline Moins de 20000 & 11,45 & 88,55 & 4,66 (3,44 à 6,33) \\
\hline \multicolumn{4}{|c|}{ Caractéristiques du mode de vie } \\
\hline \multicolumn{4}{|l|}{ Tabagisme } \\
\hline N’a jamais fumé & 3,25 & 96,75 & 1,00 \\
\hline Ex-fumeur & 6,27 & 93,73 & $1,99(1,54$ à 2,56$)$ \\
\hline Fumeur & 8,32 & 91,68 & $2,70(2,14$ à 3,40$)$ \\
\hline \multicolumn{4}{|c|}{ Caractéristiques liées à la santé } \\
\hline \multicolumn{4}{|l|}{ État de santé général } \\
\hline Excellent & 2,21 & 97,79 & 1,00 \\
\hline Très bon & 3,43 & 96,57 & $1,57(1,13$ à 2,16$)$ \\
\hline Bon & 6,20 & 93,80 & 2,92 (2,16 à 3,94) \\
\hline Assez bon & 14,36 & 85,64 & $7,41$ (5,39 à 10,17$)$ \\
\hline Mauvais & 21,94 & 78,06 & $12,41(8,88$ à 17,35$)$ \\
\hline \multicolumn{4}{|l|}{ Diabète } \\
\hline Non & 8,10 & 91,90 & 1,00 \\
\hline Oui & 13,16 & 86,84 & $1,72(1,01$ à 2,96$)$ \\
\hline \multicolumn{4}{|l|}{ IMC $\left(\mathrm{kg} / \mathrm{m}^{2}\right)$} \\
\hline$<24,9$ & 6,00 & 94,00 & 1,00 \\
\hline $25,0-29,9$ & 5,51 & 94,49 & $0,91(0,73$ à 1,13$)$ \\
\hline$>29,9$ & 7,34 & 92,66 & $1,26(1,02$ à 1,55$)$ \\
\hline
\end{tabular}

Abréviations : IC, intervalle de confiance; IMC, indice de masse corporelle; RC, rapport de cotes.

a D'après les participants à l'EAPA s'identifiant soit comme Indien de l'Amérique du Nord, Métis ou Inuit, soit comme Indien inscrit ou relevant des traités, soit comme membre d'une bande indienne, soit comme ayant une ascendance autochtone, soit une combinaison de ces réponses.

b Selon les définitions de Statistique Canada ${ }^{15}$.

c Yukon, Territoires du Nord-Ouest, Nunavut.

d Alberta, Saskatchewan, Manitoba.

e île-du-Prince-Édouard, Nouveau-Brunswick, Nouvelle-Écosse, Terre-Neuve.

IC à $95 \%$ : 1,00 à 1,10). Comme prévu, les répondants plus âgés étaient plus nombreux à faire état de $\mathrm{BC}$ que ceux du groupe d'âge le plus jeune ( $\geq 55$ ans : $\mathrm{RC}=3,06$; IC à $95 \%: 2,73$ à 3,43). Ceux qui n’avaient jamais été mariés ou qui étaient veufs ou divorcés étaient moins nombreux à faire état de BC (jamais mariés : $\mathrm{RC}=0,72$; IC à $95 \%: 0,68$ à 0,78 ; veufs/divorcés : $\mathrm{RC}=0,90$; IC à $95 \%: 0,84$ à 0,96$)$. Le revenu et le niveau d'instruction étaient inversement associés à la BC. En effet, la probabilité de BC était 1,4 fois plus élevée (IC à $95 \%$ : 1,30 à 1,57) chez les participants n'ayant pas terminé le secondaire que chez les titulaires d'un diplôme universitaire, et
3,4 fois plus élevée (IC à $95 \%$ : 3,1 à 3,6) chez ceux dont le revenu était inférieur à 20000 \$ que chez ceux ayant un revenu de 80000 \$ et plus. On a par ailleurs observé une association positive entre le fait de vivre en milieu urbain et la BC $(\mathrm{RC}=1,31$; IC à $95 \%: 1,25$ à 1,38$)$. L'IMC s'est révélé un prédicteur important comme terme quadratique, représentant une relation en $\mathrm{U}\left(\mathrm{IMC}=25,0\right.$ à $29,9 \mathrm{~kg} / \mathrm{m}^{2}$ : $\mathrm{RC}=0,91, \mathrm{IC}: 0,73$ à 1,$13 ; \mathrm{TMC}>29,9 \mathrm{~kg} / \mathrm{m}^{2}$ : $\mathrm{RC}=1,26$, IC : 1,02 à 1,55).

On a également noté deux interactions significatives entre le sexe et le tabagisme et entre le sexe et l'IMC. Chez les nonfumeurs n'ayant jamais fumé et chez les fumeurs, la probabilité de BC était plus élevée chez les femmes, tandis que chez les ex-fumeurs elle était légèrement inférieure chez les femmes (figure 1). Dans les trois catégories d'IMC (poids normal et insuffisance pondérale, surpoids et obésité), la probabilité de BC était significativement plus élevée chez les femmes, avec une différence particulièrement marquée chez les personnes obèses.

\section{Analyse}

À l'aide d'une cohorte transversale, cette étude a permis de déterminer la prévalence de la BC et d'examiner les facteurs qui y sont associés chez les Autochtones adultes. Nous avons conclu à une prévalence de la BC de 6,0 \% globalement, de 6,6 \% chez les membres des Premières nations, de 6,2 \% chez les Métis et de $2,4 \%$ chez les Inuits. L'analyse multivariée a montré que le vieillissement, le tabagisme, l'obésité, un faible niveau d'instruction, un faible revenu et la vie en milieu urbain sont associés de façon significative à la BC autodéclarée comme diagnostiquée par un médecin. Des interactions réciproques entre le sexe et le tabagisme et entre le sexe et l'IMC ont également été relevées.

Nous avons constaté que la prévalence de la BC dans notre analyse était légèrement supérieure aux valeurs de 4,9\% pour les Autochtones vivant hors réserve et de $2,4 \%$ pour la population non autochtone qui figuraient dans l'ESCC de 2005. L'ESCC mesure la BC autodéclarée comme diagnostiquée par un fournisseur de soins de santé d'une manière similaire à l'EAPA.

La prévalence de la BC s'est révélée particulièrement faible chez les Inuits par rapport aux membres des Premières nations et aux Métis. Comme c'est dans ce groupe que les taux de tabagisme étaient les plus élevés ${ }^{16}$, il faut peut-être attribuer la faible prévalence de la $\mathrm{BC}$ à des obstacles géographiques à l'accès aux soins et, donc, à des possibilités plus limitées d'obtenir un diagnostic. Cette explication pourrait aussi valoir, en partie du moins, pour la différence observée entre les lieux de résidence, les personnes vivant en milieu urbain étant plus susceptibles d'autodéclarer une BC 
TABLEAU 2

Résultats de la régression logistique de la prévalence de la bronchite chronique chez les Autochtones $^{\mathrm{a}}$ (15 ans et plus), 2006, Canada ( $\mathrm{N}=48$ 921)

\begin{tabular}{|c|c|c|}
\hline & $\begin{array}{l}\text { Estimations par régression }(\hat{\beta}) \\
\qquad \hat{\beta}(\operatorname{ET}(\hat{\beta}))\end{array}$ & $\mathrm{RC}_{\mathrm{aj} .}$ (IC à $\left.95 \%\right)$ \\
\hline \multicolumn{3}{|c|}{ Caractéristiques démographiques } \\
\hline \multicolumn{3}{|l|}{ Origine ethnique } \\
\hline Première Nation (réf.) & - & 1,00 \\
\hline Métis & $0,05(0,02)$ & $1,05(1,00$ à 1,10$)$ \\
\hline \multicolumn{3}{|l|}{ Sexe } \\
\hline Homme (réf.) & - & 1,00 \\
\hline Femme & $0,53(0,13)$ & $1,71(1,32$ à 2,21$)$ \\
\hline \multicolumn{3}{|l|}{ Âge (années) } \\
\hline 15-19 (réf.) & - & 1,00 \\
\hline $20-24$ & $0,08(0,06)$ & $1,08(0,95$ à 1,23$)$ \\
\hline $25-34$ & $0,08(0,06)$ & $1,08(0,96$ à 1,21$)$ \\
\hline $35-44$ & $0,65(0,06)$ & $1,92(1,72$ à 2,14$)$ \\
\hline $45-54$ & $1,08(0,06)$ & $2,94(2,63$ à 3,29) \\
\hline 55 et plus & $1,12(0,06)$ & $3,06(2,73$ à 3,43$)$ \\
\hline \multicolumn{3}{|l|}{ Situation de famille } \\
\hline Marié (réf.) & - & 1,00 \\
\hline Jamais marié & $-0,32(0,03)$ & $0,72(0,68$ à 0,78$)$ \\
\hline Veuf/divorcé & $-0,11(0,04)$ & $0,90(0,84$ à 0,96$)$ \\
\hline \multicolumn{3}{|l|}{ Lieu de résidence $^{b}$} \\
\hline Rural (réf.) & - & 1,00 \\
\hline Urbain & $0,25(0,02)$ & $1,31(1,25$ à 1,38$)$ \\
\hline \multicolumn{3}{|l|}{ Niveau d'instruction } \\
\hline Diplôme universitaire & - & 1,00 \\
\hline Études universitaires & $0,29(0,04)$ & $1,33(1,22$ à 1,45$)$ \\
\hline Secondaire terminé & $0,09(0,05)$ & $1,09(0,99$ à 1,21$)$ \\
\hline Moins que le secondaire & $0,36(0,05)$ & $1,43(1,30$ à 1,57$)$ \\
\hline \multicolumn{3}{|l|}{ Revenu (\$) } \\
\hline 80000 et plus (réf) & - & 1,00 \\
\hline 60 000-79 999 & $0,66(0,04)$ & $1,94(1,79$ à 2,10$)$ \\
\hline 40 000-59 999 & $0,66(0,04)$ & $1,93(1,78$ à 2,08$)$ \\
\hline 20 000-39 999 & $0,76(0,04)$ & $2,14(1,98$ à 2,31$)$ \\
\hline Moins de 20000 & $1,21(0,04)$ & $3,36(3,11$ à 3,63$)$ \\
\hline IMC $\left(\mathrm{kg} / \mathrm{m}^{2}\right)$ & $-0,07(0,01)$ & $0,93(0,91$ à 0,95$)$ \\
\hline $\mathrm{IMC}^{2}$ & $0,00(0,00)$ & $1,00(1,00$ à 1,00$)$ \\
\hline \multicolumn{3}{|l|}{ Tabagisme } \\
\hline N’a jamais fumé (réf) & - & 1,00 \\
\hline Ex-fumeur & $0,78(0,07)$ & $2,19(1,91$ à 2,50$)$ \\
\hline Fumeur & $1,18(0,06)$ & $3,24(2,86$ à 3,67$)$ \\
\hline
\end{tabular}

Suite page suivante

diagnostiquée par un médecin que celles vivant en milieu rural.

Nos conclusions sur les différences entre les sexes en ce qui concerne la prévalence de la BC sont corroborées par une étude réalisée dans une petite ville de la Saskatchewan et portant sur une population de producteurs de céréales : elle conclut à une prévalence de 9,6\% chez les femmes et de $4,2 \%$ chez les hommes $^{17}$.
De nombreuses autres études concluent également que le tabagisme, le revenu et un faible niveau d'instruction sont associés de manière indépendante à la $\mathrm{BC}^{5,18,19}$. Le tabagisme est un facteur de risque majeur et connu de la $\mathrm{BC}^{19}$. Le revenu et l'instruction, indicateurs du statut socioéconomique, donnent à penser que d'autres variables interviennent peutêtre dans cette association ${ }^{18}$. Un faible revenu, par exemple, limite les options individuelles en ce qui a trait à l'environnement, à des habitudes de vie et à une alimentation saines, ce qui peut contribuer à l'obésité ${ }^{20}$.

Le lien entre obésité et maladies respiratoires chroniques est de plus en plus reconnu. Dans une cohorte longitudinale, Guerra et collab. $^{21}$ ont constaté que les patients souffrant de BC sont plus nombreux à être obèses. Dans notre étude, nous avons observé une tendance de risque en $U$ possible (figure 1), ce qui veut dire qu'il existe une corrélation avec la maladie, que l'IMC soit faible ou élevé. Guerra et collab. ${ }^{21}$ relèvent une tendance similaire, quoique non significative. De plus, ils observent une relation temporelle. Autrement dit, un IMC de $28 \mathrm{~kg} / \mathrm{m}^{2}$ ou plus fait augmenter le risque de diagnostic de $\mathrm{BC}$ confirmé par un médecin $(\mathrm{RC}=1,80$; IC à $95 \%: 1,32$ à 2,46) deux ans plus tard ${ }^{21}$. Leur étude laisse supposer un lien de causalité, mais d'autres recherches sont nécessaires pour élucider ce lien. Quoi qu'il en soit, l'obésité augmente le risque de dysfonction respiratoire, comme l'indique une étude sur l'obésité ${ }^{22}$.

\section{Limites}

Notre étude comportait plusieurs limites. Dans des enquêtes telles que l'EAPA, l'évaluation de la BC manque de précision clinique, ce qui pourrait entraîner des erreurs de classification ${ }^{23}$. L'EAPA pose une seule question au sujet de la $\mathrm{BC}$, à savoir si un professionnel de la santé a dit aux répondants qu'ils souffraient de BC. Le diagnostic de maladie chronique peut également dépendre de l'existence et de l'utilisation des services de santé, d'où un biais systémique possible. De plus, toutes les réponses à cette enquête étaient autodéclarées, et les autodéclarations peuvent 
TABLEAU 2 (Suite)

Résultats de la régression logistique de la prévalence de la bronchite chronique chez les Autochtones $^{\mathrm{a}}$ (15 ans et plus), 2006, Canada ( $\mathrm{N}=48$ 921)

\begin{tabular}{lcc} 
& $\begin{array}{c}\text { Estimations par régression }(\hat{\beta}) \\
\hat{\beta}(\operatorname{ET}(\hat{\beta}))\end{array}$ & $\mathbf{R C}_{\mathbf{a j} .}(\mathrm{IC}$ à $95 \%)$ \\
\hline $\begin{array}{l}\text { Interactions } \\
\text { (Sexe plus tabagisme) } \\
\quad \text { Femme plus ex-fumeuse }\end{array}$ & $-1,01(0,08)$ & $0,36(0,31$ à 0,43$)$ \\
$\quad$ Femme plus fumeuse & $-0,60(0,07)$ & $0,55(0,48$ à 0,63$)$ \\
(Sexe plus IMC) & $0,01(0,00)$ & $1,01(1,00$ à 1,02$)$ \\
\hline Femme plus IMC & & \\
\hline
\end{tabular}

Abréviations : ET, écart-type; IC, intervalle de confiance; IMC, indice de masse corporelle; $\mathrm{RC}_{\mathrm{aj}}$, rapport de cotes ajusté.

a D'après les participants à l'EAPA s'identifiant soit comme Indien de l'Amérique du Nord, Métis ou Inuit, soit comme Indien inscrit ou relevant des traités, soit comme membre d'une bande indienne, soit comme ayant une ascendance autochtone, soit une combinaison de ces réponses.

b Selon les définitions de Statistique Canada ${ }^{15}$.

sous-évaluer la prévalence de certains facteurs de risque, comme le poids, le tabagisme et le revenu. Enfin, cette enquête n'a recueilli des données que sur les membres des Premières nations vivant hors réserve. Or, d’après le recensement de 2006, environ $40 \%$ des membres des Premières nations vivent dans des réserves ${ }^{14}$ et diverses statistiques révèlent des différences significatives entre les membres des Premières nations vivant dans les réserves et ceux vivant hors réserve, ce qui fait que ces résultats ne sont pas forcément généralisables à toutes les Premières nations. En outre, les Inuits ont été exclus de l'analyse multivariée, ce qui rend ces conclusions encore moins généralisables à cette population.

\section{Conclusion}

À notre connaissance, il s'agit du premier rapport examinant spécifiquement les facteurs associés à la BC chez les Autochtones. Notre recherche fournit un instantané de la BC et de ses déterminants, mais d'autres analyses sont nécessaires pour étudier de plus près ces associations, en particulier l'incidence d'un statut socioéconomique faible et de l'obésité sur la BC. Notre étude souligne l'importance du renoncement au tabagisme et de

FIGURE 1

Graphique avec barres d'erreur indiquant la probabilité de bronchite chronique chez les Autochtones (15 ans et plus), par sexe et par catégorie de tabagisme, 2006, Canada

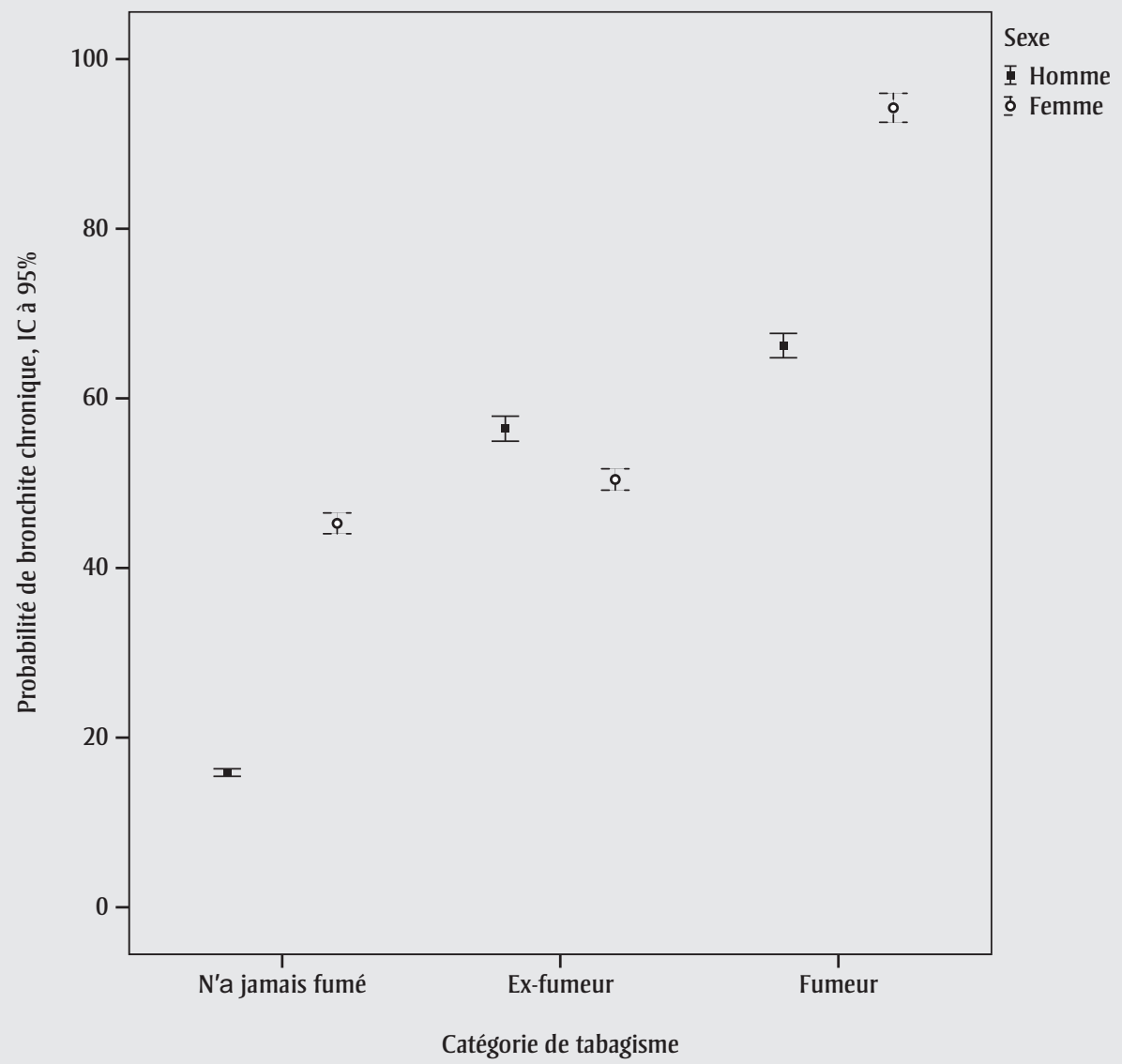


FIGURE 2

Graphique avec barres d'erreur indiquant la probabilité de bronchite chronique chez les Autochtones (15 ans et plus), par sexe et indice de masse corporelle, 2006, Canada

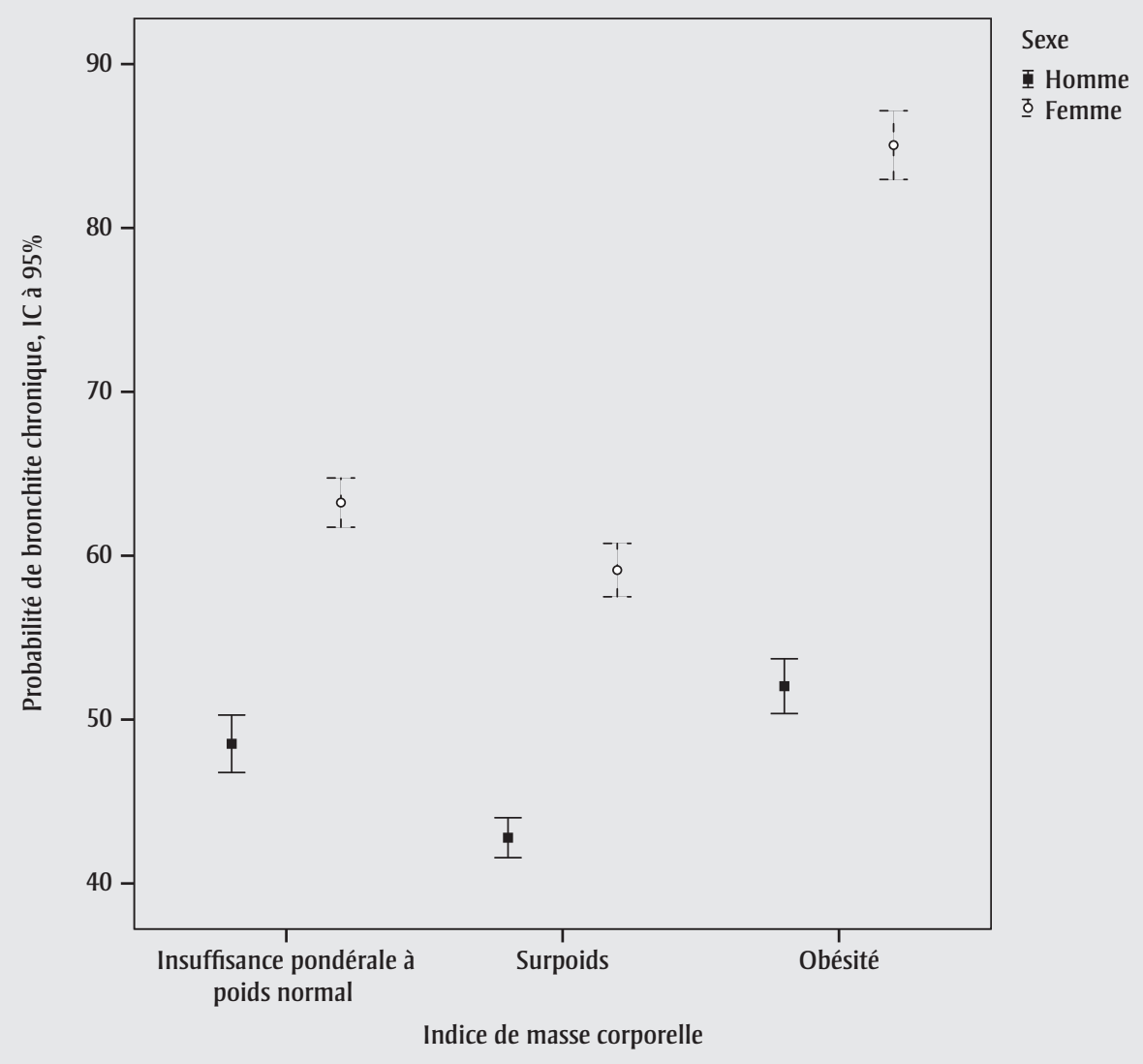

la réduction de l'IMC dans cette population, en particulier chez les femmes.

En conclusion, cette étude montre que des facteurs de risque évitables (statut socioéconomique faible, obésité et tabagisme) sont associés de façon significative à la $\mathrm{BC}$ après ajustement pour les variables confusionnelles éventuelles. Ces données peuvent être utiles dans la conception et la promotion de campagnes de prévention axées sur la population autochtone.

\section{Remerciements}

Cette étude a reçu l'appui du programme de projets pilotes des Instituts de recherche en santé du Canada et du Centre canadien de santé et sécurité en milieu agricole.

Aucun conflit d’intérêts n’est à déclarer.

\section{Références}

1. MacMillan HL, MacMillan AB, Offord DR, Dingle JL. Aboriginal Health. CMAJ. 1996;155:1569-78.

2. Sin DD, Wells H, Svenson LW, Man SF. Asthma and COPD among Aboriginals in Alberta, Canada. Chest. 2002;121:1841-6.

3. Statistique Canada. Enquête sur la santé dans les collectivités canadiennes (ESCC) : information détaillée pour 2005 (cycle 3.1) [Internet]. Ottawa (Ont.) : Statistique Canada; [modification le 10 oct. 2007; consultation le $1^{\mathrm{er}}$ juin 2010]. Consultable en ligne à la page : http://www23.statcan .gc.ca/imdb/p2SV_f.pl?Function = getSurvey \&SurvId $=3226 \&$ SurvVer $=0$ \&InstaId $=15282$ \&InstaVer $=3 \&$ SDDS $=3226 \& l a n g=e n \& d b=$ imdb\&adm $=8 \&$ dis $=2$
4. Santé Canada. Profil statistique de la santé des Premières nations au Canada : utilisation des services de santé dans l'Ouest canadien, 2000 [Internet]. Ottawa (Ont.) : Santé Canada; 2009 [consultation le $1^{\text {er }}$ juin 2010]. Consultable en ligne à la page : http://www.hc-sc.gc.ca/fniah-spnia/pubs /aborig-autoch/2009-stats-profil-vol2/index -fra.php

5. American Thoracic Society. Definitions and classifications of chronic bronchitis, asthma and pulmonary emphysema. Am Rev Respir Dis. 1962;85:762-8.

6. Pelkonen M. Smoking: relationship to chronic bronchitis, chronic obstructive pulmonary disease and mortality. Curr Opin Pulm Med. 2008;14:105-9. 
7. First Nations Information Governance Committee. First Nations Regional Longitudinal Health Survey (RHS) 2002/ 03: results for adults, youth and children living in First Nations communities. Ottawa (Ont.): First Nations Centre; Nov 2005.

8. Minore B, Hill ME, Park J et collab. Understanding respiratory conditions among Ontario's Aboriginal population. Thunder Bay (Ont.) : Centre for Rural and Northern Health Research; 2010.

9. Melia RJ, Chinn S, Rona RJ. Respiratory illness and home environment of ethnic groups. Br Med J. 1988;296:1438-41.

10. Cooreman J, Redon S, Levallois M, Liard R, Perdrizet S. Respiratory history during infancy and childhood, and respiratory conditions in adulthood. Int J Epidemiol. 1990;19:621-7.

11. Wong S. Use and misuse of tobacco among Aboriginal peoples. Paediatr Child Health. 2006;11(10):681-5.

12. Statistique Canada. Un aperçu des statistiques sur les Autochtones. Revenu [Internet]. Ottawa (Ont.) : Statistique Canada; 2010 [consultation le 22 juillet 2010]. Consultable en ligne à la page : http://www.statcan.gc.ca/pub/89-645-x /2010001/income-revenu-fra.htm

13. Statistique Canada. Portrait de la scolarité au Canada, Recensement de 2006 [Internet]. Ottawa (Ont.) : Statistique Canada; 2008 [consultation le 22 juillet 2010]. Consultable à partir de la page : http: //www12.statcan.gc.ca/census-recensement /2006/as-sa/97-560/index-fra.cfm

14. Statistique Canada. Peuples autochtones du Canada en 2006 : Inuits, Métis et Premières nations, Recensement de 2006 [Internet]. Ottawa (Ont.) : Statistique Canada; 2008 [consultation le 20 juillet 2010]. PDF téléchargeable à partir de la page : http: //www12.statcan.ca/census-recensement /2006/as-sa/97-558/pdf/97-558-XIF2006001 .pdf

15. Enquête auprès des peuples autochtones (EAPA) [Internet]. Ottawa (Ont.) : Statistique Canada; 2009 [consultation le 22 juillet 2010]. Consultable en ligne à la page: http://www.statcan.gc.ca/imdb-bmdi /3250-fra.htm
16. Hare J. Aboriginal women and healthcare. Friends of women and children in B.C. Report Card, (2004). 3(12).

17. Chen Y, Horne SL, McDuffie HH, Dosman JA. Combined effect of grain farming and smoking on lung function and the prevalence of chronic bronchitis. Int J Epidemiol. 1991;20(2):416-23.

18. Menezes AM, Victora CG, Rigatto M. Prevalence and risk factors for chronic bronchitis in Pelotas, RS, Brazil: a population based study. Thorax. 1994;49:1217-21.

19. Sethi JM, Roschester CL. Smoking and chronic obstructive pulmonary disease. Clin Chest Med. 2000;21:67-86.

20. Statistique Canada. Obésité au Canada. Déterminants et facteurs contributifs [Internet]. Ottawa (Ont.): Statistique Canada; 2010 [consultation le 20 juillet 2010]. Consultable en ligne à la page : http://www.phac-aspc.gc.ca/hp-ps/hl-mvs /oic-oac/determ-fra.php

21. Guerra S, Sherrill DL, Bobadilla A, Martinez $\mathrm{FD}$, Barbee RA. The relation of body mass index to asthma, chronic bronchitis, and emphysema. Chest. 2002;122:1256-63.

22. Poulain M, Doucet M, Major GC et collab. The effect of obesity on chronic respiratory diseases: pathophysiology and therapeutic strategies. CMAJ. 2006;174(9):1293-9.

23. Bobadilla A, Guerra S, Sherrill D, Barbee R. How accurate is the self-reported diagnosis of chronic bronchitis? Chest. 2002;122: 1234-9. 